Document downloaded from:

http://hdl.handle.net/10251/145860

This paper must be cited as:

Jimenez, JM.; Romero Martínez, JO.; Lloret, J.; Díaz Santos, JR. (15-0). Energy Savings Consumption on Public Wireless Networks by SDN Management. Mobile Networks and Applications. 24(2):667-677. https://doi.org/10.1007/s11036-016-0784-7

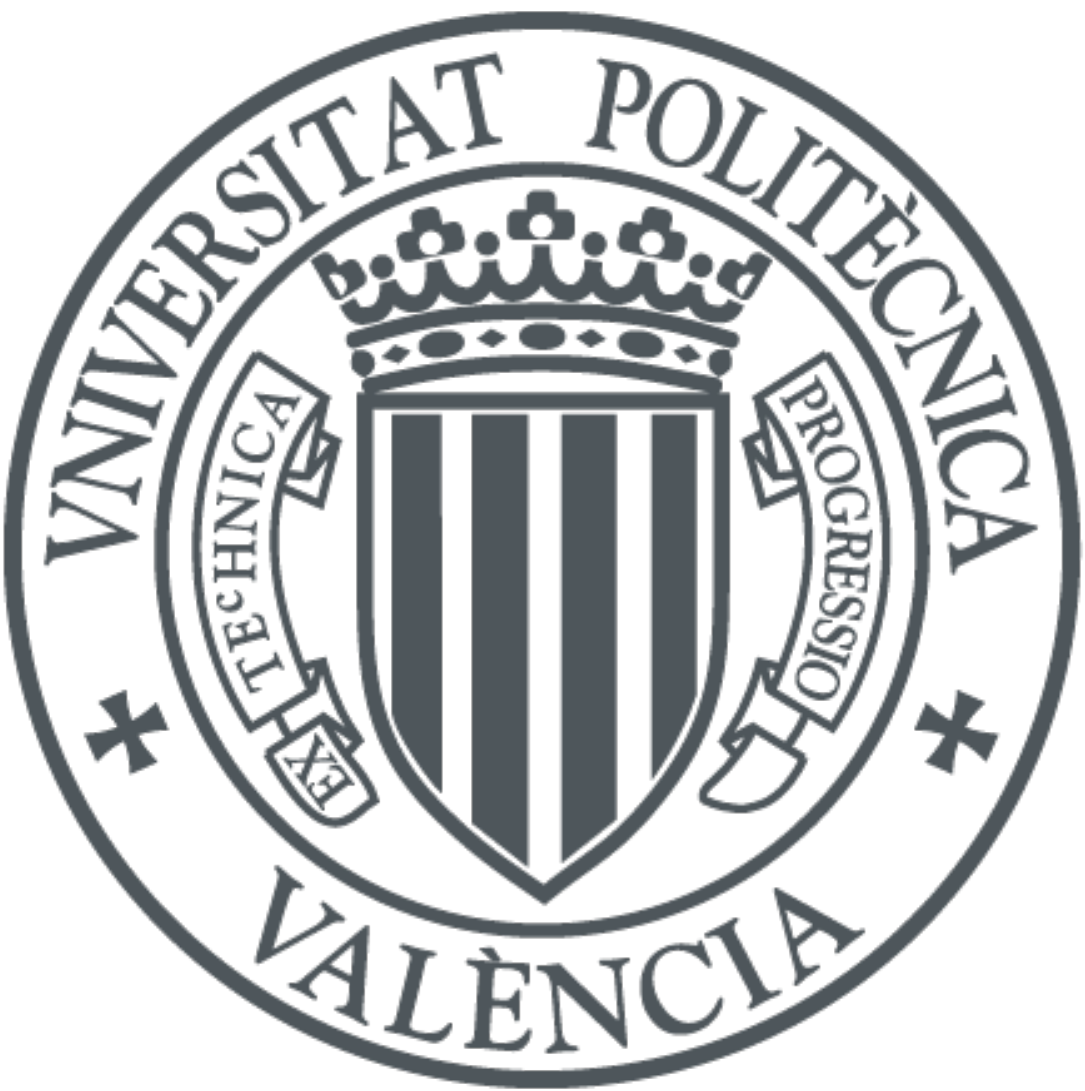

The final publication is available at

https://doi.org/10.1007/s11036-016-0784-7

Copyright Springer-Verlag

Additional Information 


\title{
Energy Savings Consumption on Public Wireless Networks by SDN Management
}

\author{
Jose M. Jimenez, Oscar Romero, Jaime Lloret and Juan R. Diaz \\ Universidad Politécnica de Valencia \\ Camino Vera s/n 46022, Valencia (Spain) \\ jojiher@dcom.upv.es, oromero@dcom.upv.es, jlloret@dcom.upv.es, juadasan@dcom.upv.es
}

\begin{abstract}
In order to use the energy more efficiently, network algorithms and protocols must incorporate in their decision mechanisms some functions focused on saving energy. In this paper we have studied different features and parameters of wireless networks to establish their relationship with the power consumption. First, we have analyzed the variation of power consumption of access points (APs) in function of the antenna transmission power. Second, we have collected user information from real public wireless network to determine the real requirements of network resources in real time basis. Based on this information, a new extension for Openflow protocol over SoftwareDefined Networking (SDN) networks will be proposed to manage the wireless network, with the aim to keep the optimal network performance with minimal power consumption. This extension introduces new Openflow messages and a new function to be incorporated in SDN controllers and Openflow enabled devices. The proposal will be validated by appropriated simulations based on real scenarios: a shopping center and a municipal wireless network.
\end{abstract}

Keywords- Energy consumption, Energy efficiency, OpenFlow, Software-Defined Network, Green Networks, Municipal Public Wireless.

\section{INTRODUCTION}

Nowadays, there is a special awareness to preserve the environment. In the past, there was not a real concern about the amount of energy consumed to keep our way of life. Significant technical advances that require high energy consumption, for both, domestic and industrial environments, have produced a real concern about the consumption of energy. This lack of energy control has a very high environmental cost, which is related to global warming and climate change. Facing with these problems, it should be adopted a series of changes, improvements and commitments to decrease energy consumption in computer networks.

Generally, electricity consumption in the operation of servers, computers and other ICT equipment represents a large part of the energy consumed by government institutions and enterprises. We need to take more and better measures to reduce the environmental impact of ICTs. The savings in energy consumption is important for any budget. As we observe every day, the cost of electricity bills increases, in future the cost of electricity bills will reach an important percentage of the total cost of ownership (TCO).

Network devices have evolved, in recent years, to improve network performance [1]. Today, many of the devices connected to the network are mobile devices. Moreover, different studies indicate that a large increase of mobile users will occur in the near future. Among these studies, we can highlight the Cisco Visual Networking Index Report [2]. This report indicates that in 2020 the total number of smart devices will be nearly 50 percent of global devices; the number of connected mobile devices per capita will reach 1.5; monthly global mobile data traffic will be 30.6 exabytes, and more traffic will be offloaded from cellular and Wi-Fi networks. Globally, total public Wi-Fi hotspots (including homespots) will grow 7-fold from 2015 to 2020, from 64.2 million in 2015 to 432.5 million by 2020. Due to this increase, networks need higher availability of Wi-Fi hotspot infrastructures. These predictions show us the need to reduce energy consumption on network devices.

Most public buildings and shopping centers have a restricted opening hours. Because of this, part of the ICT equipment is not required to be activated when there are no users in the facilities. Therefore, it can be treated this ICT equipment as if they were "vampire powers". Thus, as suggested in Climate Savers [3] that is a trademark of World Wildlife Fund (WWF) [4], we can plug all our electronics into one device, so we can switch off all of them easily when we don't use them.

If Energy Star [5] certified equipment is used, it can save energy. For example, Energy Star certified small network equipment (SNE) use 20 percent less energy than conventional models. A variety of products are covered by SNE specification: Cable and DSL modems, optical network devices, Cable and DSL integrated access devices, routers, switches and APs.

Software-Defined Networking (SDN) [6] is an emerging architecture that is dynamic, manageable, cost-effective, and adaptable, making it ideal for the high-bandwidth, dynamic nature of today's applications. This architecture decouples the network control and forwarding functions enabling the network control to become directly programmable and the underlying infrastructure to be abstracted for applications and network services.

OpenFlow [7] is a communication protocol that allows us to control network devices by managing the forwarding plane. The OpenFlow protocol is the basis of open SDN standards-based. It is the first standard communications interface of a SDN 
architecture. Using OpenFlow we can manage a network, thus, to address the energy consumption. The OpenFlow standard has been used in previous studies on both wired and wireless networks [8] [9].

The activation of APs can be started in different ways, that is, the device may have no power and therefore it is off, or it could be in a networked standby mode, which means a condition in which the equipment is able to resume a function through a remotely initiated trigger via a network connection [10].

In this paper, we have focused on saving energy in wireless networks. First, we will study features and parameters related to the power consumption in public wireless networks. Specifically, we have analyzed the antenna power transmission and the number and distribution of users around the network. These studies have been performed over real scenarios and real physical devices. The studied wireless network include shopping centers, museums and municipal public wireless networks. Second, we will introduce a new mechanism to manage the antenna transmission power and the activation and deactivation of the APs with the aim to reduce the power consumption.

Our proposal is based on a SDN centralized control system. The goal is to optimize the use of the energy through dynamic changes in the network devices.

The remaining of the paper is structured as follows: Section 2 shows previous related works. Section 3 describes the previous studies performed over real public wireless networks. In Section 4 we propose a new mechanism based on SDN to reduce the power consumption in wireless networks. The validation of our proposal is presented in Section 5. Finally, the conclusions and future works are shown in Section 6.

\section{RELATED WORK}

There are many previous works that studied different aspects of SDN. Yang et al. [11] proposed a time-aware software defined networking (TaSDN) architecture for OpenFlow-based datacenter optical networks, by introducing a time-aware service scheduling (TaSS) strategy. In our previous works [12] [13], we present a performance comparison between Mininet [14] and a real network when multimedia streams are being delivered. Moreover, we compared the experimental results obtained when a virtual network is generated by using Mininet versus a real implemented network, varying the Maximum Transmission Unit (MTU).

There are many papers studying the reduction of energy consumption in network devices. We can also find papers that study the reduction of energy consumption applied to the field of wireless transmissions. Nedevschi et al. [15] have presented the design and evaluation of two forms of power management schemes that reduce the energy consumption of networks. The first is based on putting network components to sleep during idle times. The second is based on adapting the rate of network operation to the offered workload. Feeney et al. [16] present a study about the energy consumption of wireless networks interfaces in ad-hoc networks. Andrade-Morelli et al. [17] present a comparative study of the energy consumption of several wireless network APs. Chen et al. in [18] proposed a series of strategies for achieving a "green network"; their solution is based on the correct choice of the IEEE 802.11 standard, as well as the different devices to form Wireless Networks.

Some researchers have proposed new intelligent algorithms for the MAC layer that improve the performance of the network [19] and, thus, reduce the energy consumption of the wireless devices [20].

Related to the reduction of energy consumption in switches managed by OpenFlow, some papers have been published. In [21], $\mathrm{Vu}$ et al. proposed an extension to OpenFlow switches to support different power saving modes. The extension includes OpenFlow messages and an OpenFlow Switch Controller (OSC) that is able to turn on/off switches and disable/enable ports. Kaup et al. [22] present measurements and derived power consumption models for two SDN devices.

\section{STUDY OF PUBLIC WIRELESS NETWORKS}

In this section we first analyze the relationship between wireless power transmission management and energy saving. Then, a study of utilization of public wireless infrastructures will be exposed. The aim of these previous works is to establish the parameters and features involved in power consumption and how they can be managed to achieve significant saving power. Based on this information, an SDN solution based on the OpenFlow protocol to manage wireless devices will be proposed and described in Section 3 of this paper.

\subsection{STUDY OF WIRELESS POWER TRANSMISSION MANAGEMENT FOR ENERGY SAVING.}

Our initial working hypothesis is that when the transmission power is reduced in wireless devices the energy consumption will decrease. If this assumption can be experimentally or theoretically confirmed, the basis for the development of new algorithms and protocols will be established in order to achieve most efficient wireless infrastructure deployments.

With the aim of proving the relation between saving energy and wireless power transmission, the following experimental set has been performed: two workstations were associated to the same wireless AP, IEEE $802.11 \mathrm{~g}$ compliance. Wireless interface was activated and the baseline energy consumption of the wireless devices was measured. Then, an UDP flow was established between both workstations by using a software application developed specifically to keep the bandwidth consumption stable and controlled. Over this scenario, some measurements of energy consumption of the access device has 
been performed for three experimental conditions as a function of the wireless transmission power configured at the antenna of the wireless device: $1 \mathrm{~mW}, 12.5 \mathrm{~mW}$ and $15 \mathrm{~mW}$. For each experimental condition, 80 measures have been taken, 20 for each of the four considered bandwidth used by the UDP application: 5, 10, 20, and $30 \mathrm{Mbps}$. Figure 1 shows the difference between each test performed and the energy consumption baseline. The baseline measures have the same value for each of the three experimental conditions, thus, the results comparisons between each of these conditions are valid.

Statistical analysis ANOVA was performed between the power consumption measures for different power transmission values with the same bandwidth transmission. Variance analysis was independently performed for measures at 5, 10, 20, and $30 \mathrm{Mbps}$ bandwidth transmission. The results, with a confidence interval of $95 \%$, $(\mathrm{p}=0.05$ ), allows us to confirm the initial hypothesis when the network bandwidth consumption is equal or greater than $10 \mathrm{Mbps}$. Then, we will be able to propose algorithms and protocol modifications to improve the energy consumption based on power transmission management, but the energy saving will be low.

Fig. 1.

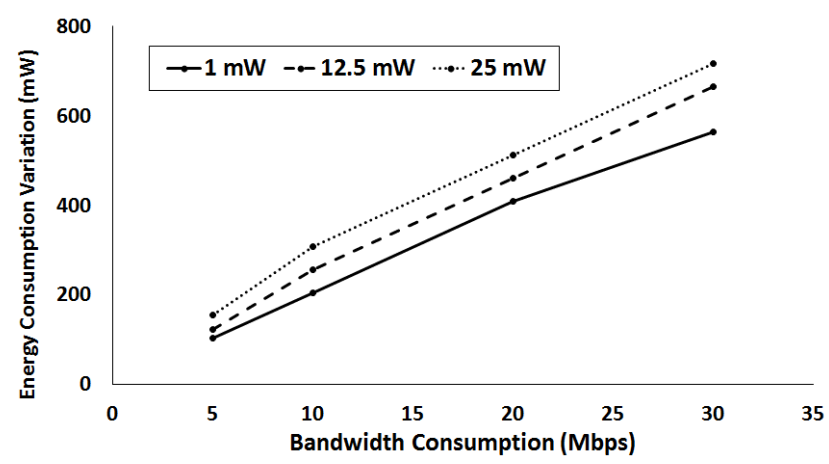

Energy consumption variation for three different wireless transmission power $(1 \mathrm{~mW}, 12.5 \mathrm{~mW}$ and $25 \mathrm{~mW})$ as a function of the consumed bandwidth by UDP user data traffic.

\subsection{STUdy OF UTILIZATION OF PUBLIC WiRELESS INFRASTRUCTURES}

Because of the increase of new Internet services that are provided on public areas, such as convention centers, stadiums, concert halls, theaters or public buildings, a study of utilization of public wireless infrastructures has been performed. The obtained results allow us to establish the effectiveness of energy saving policies that could be developed on this kind of networks.

The measurements of this study have been collected from two real public networks. The first considered network has been the wireless infrastructure in a shopping center, which is offering free Internet access to its customers. This network consists of 60 APs of Aruba manufacturer. These APs are working in IAP mode with a virtual controller. The second considered network was a municipal wireless infrastructure offering Internet access service to the citizens of a small city in public places, such as libraries, an event center and the city hall. This network consists of 40 lightweight APs of Cisco manufacturer managed by a Cisco Controller. Both networks have been designed and implemented based on the requirements to provide high quality service to a given number of users that we will call MaxUsers. For this reason, the value of MaxUsers parameter is higher than the maximum expected number of users. The value of this parameter was 800 for the shopping center and 500 for public municipal wireless. In the network design, the appropriate number of APs was selected following the MaxUsers parameter of the network and the layout of the buildings and public places where the service is provided. In order to manage the user accounts and following the local Data Protection Act, each network has implemented a captive portal by using an Aruba ClearPass server and a RAP-103 Aruba device in combination with an Aruba wireless controller. The statistics data used in this study was collected by SQL query request to the ClearPass Database. If we can demonstrate in this study that the real number of connected users is, most of the time, significantly lower than the MaxUsers, it will show us that the network is underused and power saving policies could be applied by network protocols to avoid spending unnecessary energy.

The most interesting variable for our purposes is the number of users connected at each time. Then, we could establish a direct relationship between this variable and the number of connected access devices (mainly APs) needed to provide an appropriate service to end users. Specifically, we are interested to know the variation of number of users at different hours throughout the day, and on different days along a month.

Figure 2 shows the number of users throughout of the day connected to both two real public networks described previously. First, the number of connected users has been estimated by the total number of user sessions started in every 60 minutes interval. Then, for each 60 minutes interval, the average of this number of sessions at the same hour of the day, but in different days, has been calculated in a 31 days interval (March of 2016). We can see in the figure that there are almost no users connected between 22:00 and 8:00 hours in both networks, but the APs are always connected and then the energy is still been consumed. Also, there are other specific hour intervals where the number of users is lower than the MaxUsers parameter estimated for the network. For example, the lunch time in the public municipal wireless network. Specific behaviour user patterns can be extracted for each kind of public network from these results. 


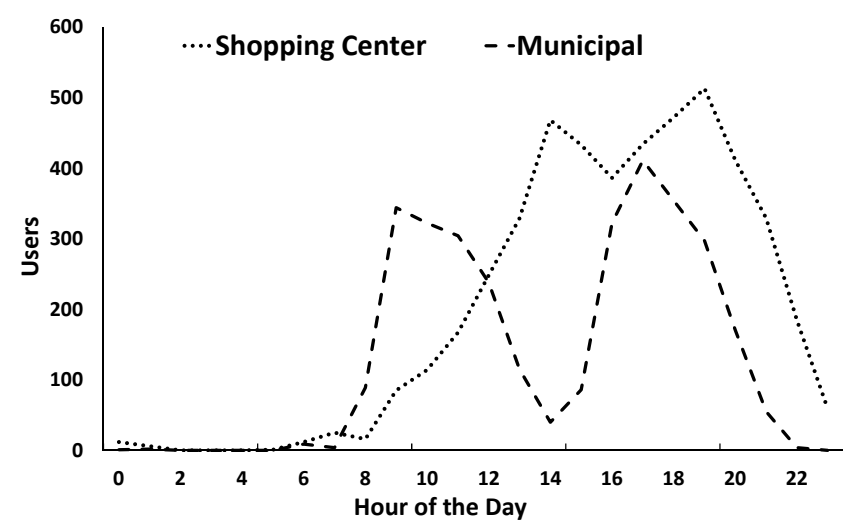

Fig. 2.

Number of users connected to two real public wireless infrastructures throughout the day.

Figure 3 shows the variation of the number of users for each day along a month (March of 2016). To perform each measurement, first the number of user sessions has been obtained by the database for each 60 minutes interval, then the average for the intervals in the same day has been calculated for each day of the month. We can see that there are some days when the network is clearly underused, for example, on Sundays $\left(6^{\text {th }}, 13^{\text {th }}, 20^{\text {th }}\right.$ and $\left.27^{\text {th }}\right)$. The shadow area shows the Easter holyday in Spain, where the studied public wireless network are located, between the $24^{\text {th }}$ and $27^{\text {th }}$. In this interval, we can see that the number of connected users is maximum in a shopping center and minimum in a municipal public wireless network. There are several days where there is a high number of users, for example, on Saturdays in the shopping center, but the amount of connected users is always lower than the MaxUsers value.

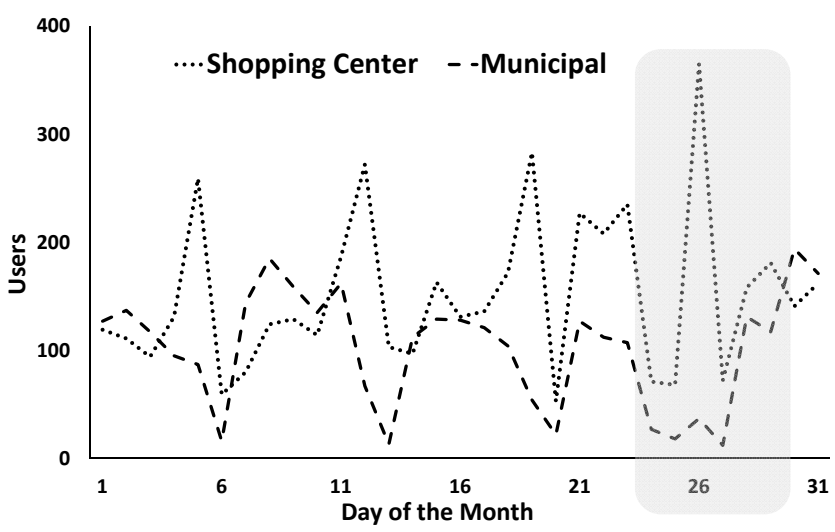

Fig. 3.

Number of users connected to two real public wireless infrastructures along the days of a month.

The results confirm that there are specific patterns for each network, and that the network resources are underused most of the time. This demonstrates that a significant amount of power saving can be achieved by introducing network protocols and algorithms, in order to control the appropriated number of connected APs. When the wireless network is underused, this network protocol will be in charge of monitoring and controlling the network devices in order to keep the same coverage area and the appropriated service level for the end users, while minimizing the power consumption.

\section{SDN MANAGEMENT SYSTEM FOR POWER SAVING}

Our proposal is based on a SDN infrastructure and the OpenFlow protocol. The centralized nature of this kind of networks gives us the necessary elements for a quick and easy deployment. Figure 4 shows the architecture of our new proposal. The SDN Controller is included with the aim of controlling dynamically the wireless devices according to the network conditions. For this purpose, new functions will be incorporated to the SDN system based on the results of the previous studies. Specifically, these functions will allow managing the antenna power transmission and the state of the devices. In order to exchange the necessary information between the SDN Controller and the wireless devices, the Openflow protocol will be extended with new messages and procedures.

First, we will present the new proposed method for saving power in wireless networks. Then, we describe the messages exchanged by the SDN Controller and the APs to configure the transmission power of the APs. 


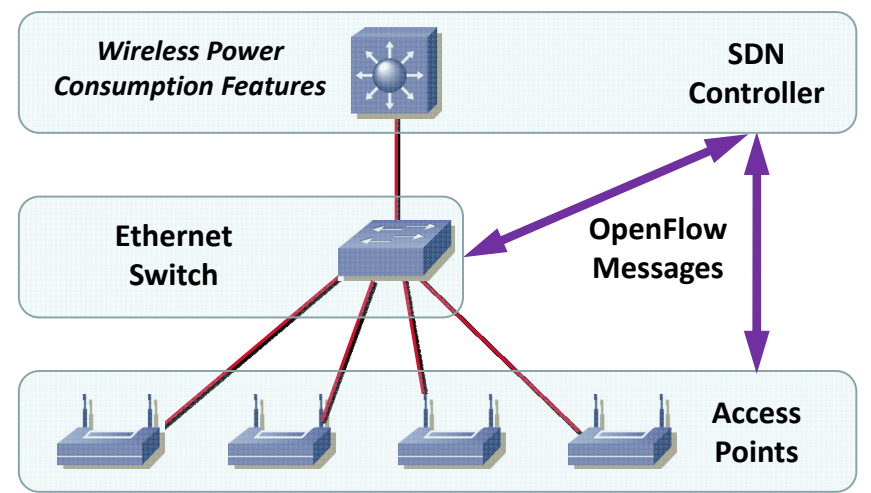

Fig. 4.

Architecture components on SDN networks used to implement new power saving features.

Figure 5 shows the OpenFlow messages exchanged by the SDN Controller and the APs. First, the SDN Controller sends an OpenFlow message to the AP in order to know the transmission power used by the AP to communicate with its clients (Figure 5, OFWP Request). Depending on the value of this parameter, the AP may need to send a test frame to some of its clients (Figure 5, Test message) to update the internal AP information. When the AP receives the answer from its clients or if this information was already updated, then the AP will send an OpenFlow message to the Controller (Figure 5, OFWP Reply).

Next, the SDN Controller will send an OPWPM Request to the AP with the transmission power to be used by the AP when sending traffic to a specific client. The transmission power of the AP could be changed for each client. Prior to be sent this OpenFlow message by the SDN Controller, there are several parameters that have been analyzed by the SDN Controller in order to calculate the optimal value of the transmission power for each AP. The most important factor in this calculation will be the relation between the QoS requirements of the client traffic and the received power from this client associated to the AP. Depending on the calculated transmission power, the AP may need to send a message in order to make a reservation of the channel to avoid the hidden station problem. Finally, the AP will confirm the used transmission power to the SDN Controller through the OFWPM Reply message.

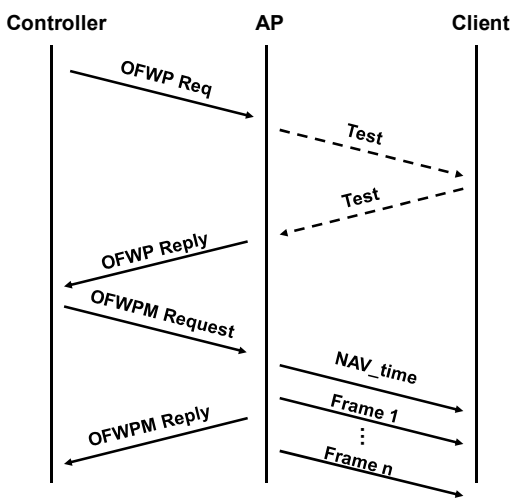

Fig. 5.

Exchange messages diagram between SDN Controller, APs and clients.

The set of the new proposed OpenFlow messages are shown in Table 1.

Table 1.

OpenFlow AP transmission power management messages and Functions

\begin{tabular}{ll}
\hline Type of message & \multicolumn{1}{c}{ Function } \\
\hline OFWP Request & Controller asks to AP client parameters \\
OFWP Reply & AP provides to Controller client parameters \\
OFWPM Request & Controlers request to AP transmit power \\
OFWPM Reply & AP confirms to Controller transmit power \\
\hline
\end{tabular}

Next, the code of the proposed OpenFlow messages is shown in Figure 6. 


\begin{tabular}{|c|c|}
\hline /* OFWP Request */ & /* OFWP Reply */ \\
\hline$I^{*}$ (OpenFlow Wfi Power Request) */ & $I^{*}$ (OpenFlow Wfi Power Reply)*/ \\
\hline $\begin{array}{l}\text { / Controller to AP message */ } \\
\text { struct ofp_wifi_pwr_mngmt \{ } \\
\text { struct ofp header header; } \\
\text { uint8_t hw_addr[OFP_ETH_ALEN]; } \\
\text { uint8_t pad[2]; }\end{array}$ & $\begin{array}{l}\text { /*AP to Controller Message */ } \\
\text { struct ofp_wifi_pwr_mngmt \{ } \\
\text { struct ofp header header; } \\
\text { uint8_t hw_addr[OFP_ETH_ALEN]; } \\
\text { uint16_t power; }\end{array}$ \\
\hline$\}$ & \} \\
\hline /* OFWPM Request */ & /* OFWPM Reply */ \\
\hline$I^{*}$ (OpenFlow Wfi Power Management Request) */ & $I^{*}$ (OpenFlow Wfi Power Management Reply) */ \\
\hline $\begin{array}{l}/^{*} \text { Controller to AP message } /^{*} \\
\text { struct ofp_wifi_pwr_mngmt }\{ \\
\text { struct ofp header header; } \\
\text { uint8_t hw_addr[OFP_ETH_ALEN]; } \\
\text { uint16_t tx_power; } \\
\text { uint16_t NAV_time; } \\
\text { uint16_t num_packets }\end{array}$ & $\begin{array}{l}\text { /*AP to Controller Message */ } \\
\text { struct ofp_wifi_pwr_mngmt }\{ \\
\text { struct ofp header header; } \\
\text { uint8_t hw_addr[OFP_ETH_ALEN]; } \\
\text { uint16_t tx_power; } \\
\text { uint16_t NAV_time; } \\
\text { uint16_t num_packets }\end{array}$ \\
\hline$\}$ & \} \\
\hline
\end{tabular}

Fig. 6.

Openflow messages for the new proposal.

Previous OpenFlow messages use the OpenFlow Header struct, showed in Figure 7.

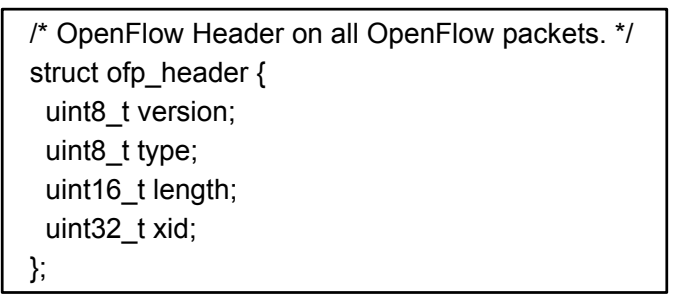

Fig. 7.

OpenFlow Header structure.

In our proposed method, the energy saving is based on the power transmission level and the status ON/OFF of the APs. Their values depend on the number of users and the average bandwidth provided for the users. These two parameters, power transmission and status ON/OFF, are independent and they provided two different power saving mechanisms. They are implemented at the same SDN Controller. Turning ON/OFF the APs points provides a high energy saving. This feature can be easily implemented by connecting the APs to Power Over Ethernet (PoE) compatible Ethernet swith ports. OpenFlow messages are sent from the SDN Controller to the switch to turn on/off the switchport where the AP is connected. In addition, to improve the power saving and the coverage area, the power transmission level of the APs can be modified by sending the new proposed OpenFlow messages.

To explain how our proposal works, we will consider a wireless network over a 2-Dimension coverage area. The whole coverage area is divided in cells. In figure 8, the coverage area has been divided in 6 cells labeled from A to F. This figure shows also the three types of APs defined in the proposed system: main, primary and secondary.

- Main AP. They are always in mode ON and at maximum transmission power. They are located in the border between several cells and provide partial wireless coverage for these cells simultaneously. The goal of this type of AP is not to provide full coverage for these cells, but to provide basic and permanent service at the center of the area. In Figure 8 we can see how the Main AP provides partial coverage to four cells (A, B, D and E).

- $\quad$ Primary AP. They are always in mode ON and, by default, they are at maximum transmission power. Every cell has a unique Primary AP in the center that provides full coverage. If the amount of users inside the cell is lower than the defined threshold, the Primary AP is configured at maximum transmission power and it provides the required service for all users in the cell. In Figure 8 we can see how the Primary AP provides full coverage to the cell B.

- Secondary AP. By default they are in mode OFF. The quantity of Secondary APs in each cell will depend on the cell position into the area and the sizing of the network. The Secondary AP will be started when the quantity of users in its cell rises the defined threshold. Then, the transmission power of Primary and Secondary APs in this cell will be managed depending on the number of users inside the cell, as explained later. In figure 8, the Secondary AP in the Cell A provides partial coverage to this cell. 
The total number of actives APs in the area will depend on the number of users and how they are placed in the different cells. When the users in a cell rises the threshold, the Secondary APs will be activated and the power transmission of the Primary and Secondary APs in the cell will be adjusted. The threshold parameter is related to the minimum bandwidth required by user.

The channels distribution configured in the APs has been selected to minimize the radio interferences. The Main APs are distributed in such way that provides coverage to the whole area.

With the aim to minimize the power consumption, by default, the Primary APs will transmit at maximum power level, in order to require the minimum number of active APs in the network. An AP transmitting at maximum power level will consume more energy that transmitting at minimum power level, but we get the highest energy saving by having the Secondary APs turned OFF. Figure 9 show this Minimum configuration, that is when the lowest number of APs are active.

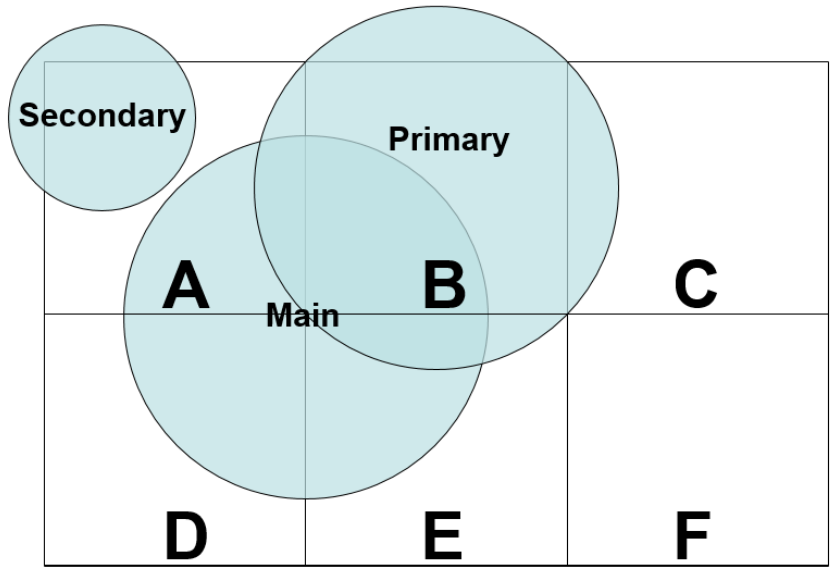

Fig. 8.

Types of APs

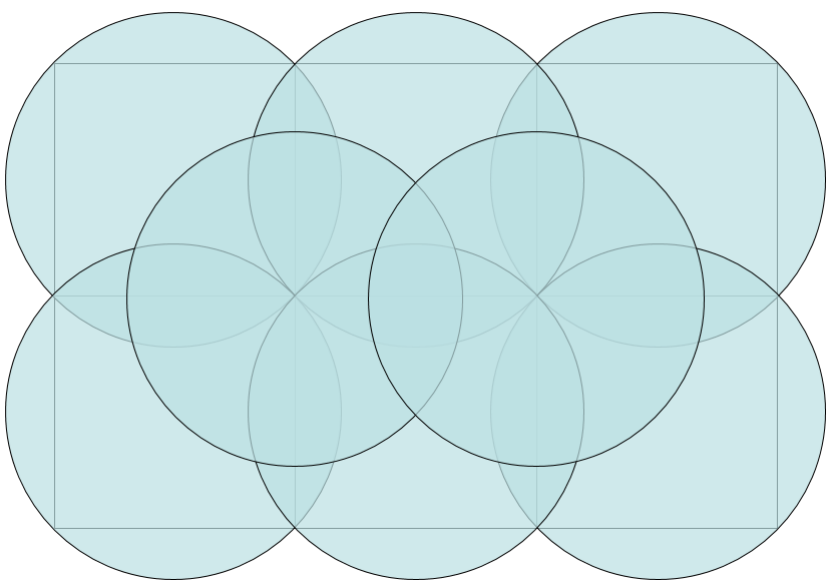

Fig. 9.

Minimum APs arrangement.

When necessary, the Primary APs could be even turned OFF at specific time intervals when there are no users expected. For example, following the results in the studies performed, at night in the shopping centers or on holiday in buildings offering public wireless services.

When the number of users increases, the SDN Controller detects it has exceed a threshold in some cell, and then it will act over the APs in that cell. First, the information about the account of connected users is sent to the SDN Controller by the Wireless Controller. Then, the SDN Controller will send an OpenFlow message to the Primary AP to decrease its transmission power level. Simultaneously, another OpenFlow messages will be sent to the PoE Ethernet switches that provides power to Secondary APs in that cell to put them in state ON. With this Medium configuration, all Primary and Secondary APs in this cell will transmit at the lowest transmission power. Figure 10 shows the Medium configuration in cell A (top-left cell in the figure). Power consumption in this cell will increase because the secondary APs are now turned ON. An advantage of this configuration is that the APs in the cell are not working at maximum power transmission, meaning a small amount of energy to be saved, as it has been demonstrated previously. Also, another advantage of not transmitting at maximum power level is to reduce the overlapping of coverage area, minimizing the interferences among the APs in that cell and also with APs in the other cells. Later, when the Controller detects that the number of connected users decreases below the threshold, it will send the corresponding OpenFlow messages to the primary AP to increase its transmission power and to the PoE Ethernet switches to disable the Ethernet ports where the secondary APs are connected.

Figure 11 shows the Maximum configuration when all wireless APs in the area are active. This happens when the number of users exceeds the threshold in all the cells. The Main AP is configured to transmit at maximum power and the remaining APs are configured at minimum power transmission. If the SDN access devices do not support the OpenFlow messages to switch between maximum and minimum transmission power, then the Primary APs keep transmitting at maximum power. The secondary APs always transmit at minimum power when they are in mode ON.

Figure 12 shows the flow diagram for the procedure to turn ON/OFF the Secondary APs. The system can modify the threshold parameter dynamically to achieve a balance between the bandwidth provided per user and the power consumption. The main goal is to provide an adequate quality in the provided services and, at the same time, to save the maximum energy as possible.

As show in figure 12, the process starts collecting information from the Wireless Controller. In our proposal the Wireless Controller will be external to our system and will be implemented by specific manufacturer solutions, such as Cisco WLC Controller or Aruba Mobility Controller. The SDN Controller compares the number of users in each cell $\left(u_{i}\right)$ with the value of the established threshold in this cell $\left(\mathrm{Th}_{\mathrm{i}}\right)$. If $u_{i}<T h_{i}$ then, the system checks if the Secondary APs in this cell are ON. If the 
APs are ON, then the SDN Controller changes the state to OFF, if the Secondary APs were already in OFF state, it will remain in this state. By the other hand, when $u_{i}>T h_{i}$ then the system checks if the Secondary APs are in OFF state. If this condition is accomplished, then the SDN Controller changes the state to ON, but in the case that the condition is not accomplished, the Secondary APs remain in the original state. The Devices Control mechanism receives the information about the state which the devices belong. Later, the Devices Control sends OpenFlow messages to network devices to establish the optimal configuration and, at the same time, a timer is activated to schedule new request information for the Wireless Controller.

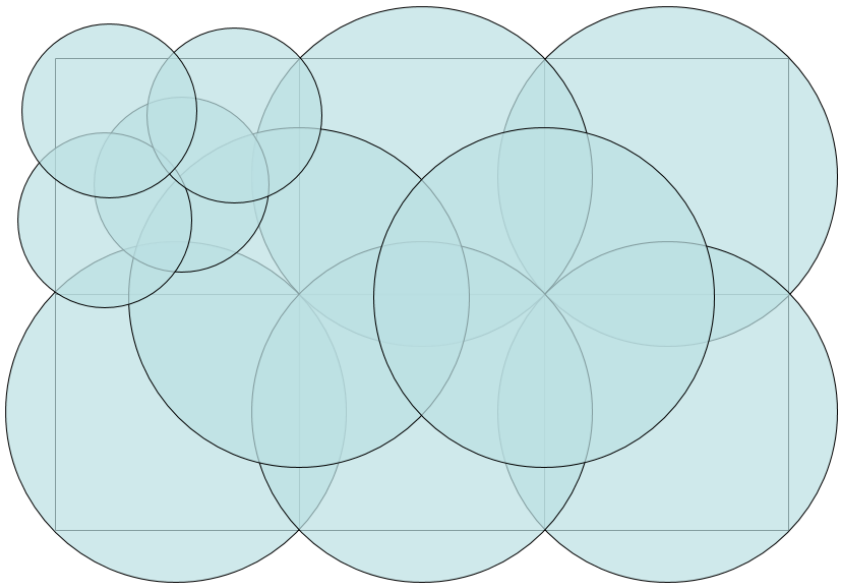

Fig. 10.

Medium APs arrangement.

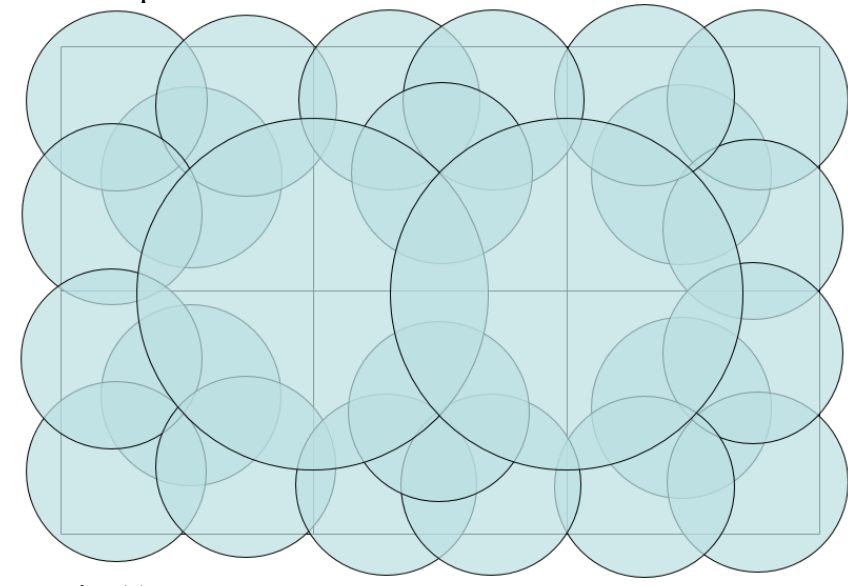

Fig. 11.

Maximum APs arrangement.

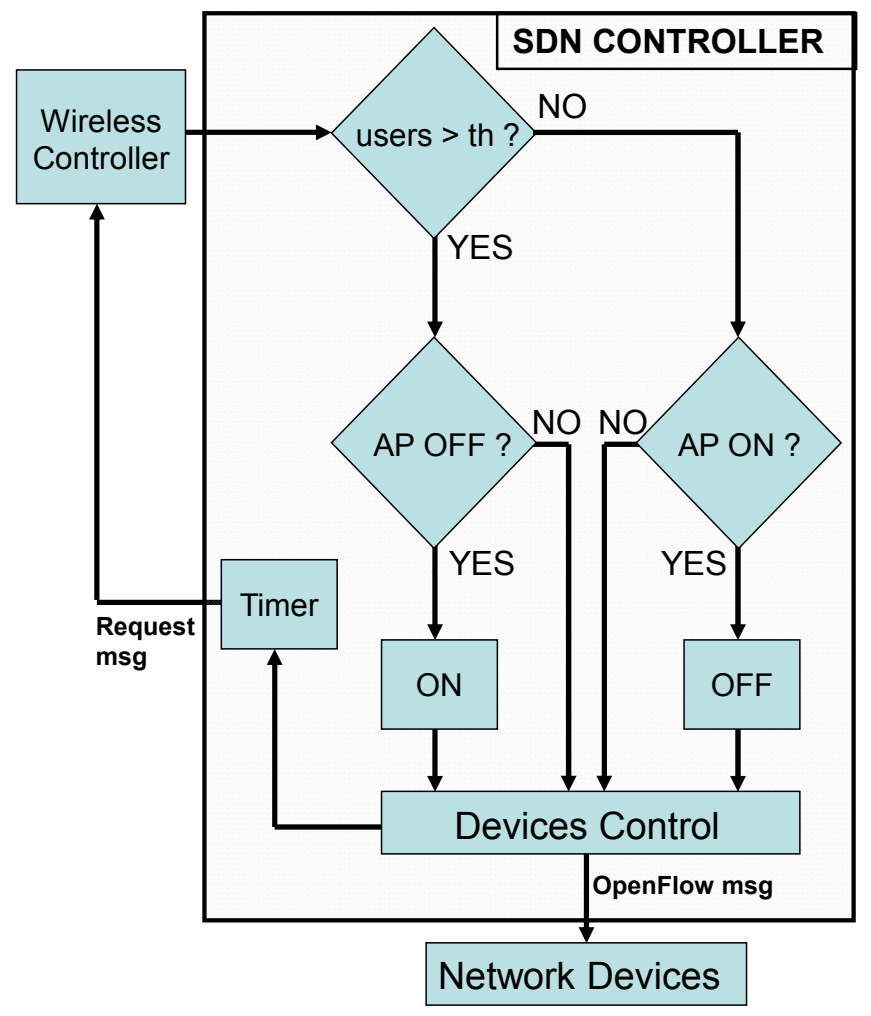

Fig. 12.

Flow diagram: Secondary APs mode ON/OFF for power saving

The calculation of the total power consumption by all APs in the topology is obtained by the addition of the power consumed by each device. The equation 1 shows this calculation: 


$$
\mathrm{P}=\sum_{\mathrm{i}, \mathrm{j}} \mathrm{P}_{\mathrm{ij}}
$$

Depending on the type of AP and the number of users in every cell, an AP will be in mode ON or OFF. When it is in mode $\mathrm{ON}$, the AP may transmit at maximum or minimum power. All possibilities are expressed at the equation 2:

Where:

$$
P_{i j}=\left\{\begin{array}{cll}
0 & , u_{i} \leq t h_{i}, & N_{i j} \text { sec } \\
P_{\min } & , u_{i}>t h_{i}, & N_{i j} \text { sec } \\
P_{\max } & , u_{i} \leq t h_{i}, & N_{i j} \text { pri } \\
P_{\min } & , u_{i}>t h_{i}, & N_{i j} \text { pri }
\end{array}\right.
$$

$P_{i j}$ : power consumed by access point $\mathrm{N}_{\mathrm{ij}}$ in cell $\mathrm{i}$

$N_{i j}(i=j)$ : primary access point

$N_{i j}(i \neq j)$ : secondary access point

$i=1,2, \ldots, C C$ :number of cells

$j=1,2, \ldots, S_{\mathrm{i}} S_{i}$ :number of secondary APs in cell $\mathrm{i}$

$u_{i}$ : number of users in cell $\mathrm{i}$

$t h_{i}$ : threshold in cell $\mathrm{i}$

Figure 13 shows the structure used for the OpenFlow header used to turn ON/OF a POE port of an Ethernet Switch.

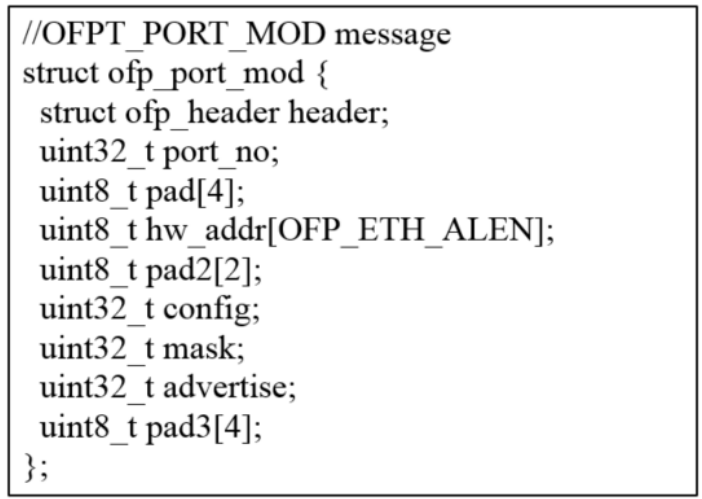

Fig. 13.

OpenFlow message used to turn ON/OFF a POE port of an Ethernet Switch.

\section{SySTEM VALIDATION}

A mathematical model, following the description of the SDN system and the results obtained in our previous studies about shopping center and municipal wireless networks, described in previous sections, will be used in this section to validate our proposal.

In this model we used the maximum power consumption values of different APs. Table 2 shows the power consumption of several models of APs from different manufacturer, such as Aruba, Cisco and Aerohive. All values related to the power consumed by the APs are provided by the manufacturers themselves in their data sheet [23-30]. These AP models have been used because they are also used in the real public environments that we have studied previously, so the results of simulations could be translated to these real networks directly. All AP models considered are powered by PoE. The typical power consumption of an AP is over 10 Watts. Even, for most of them, the power consumption is over 15 Watts.

Figure 14 shows the users who have visited a real shopping center for a period of one day ( 24 hours). The figure shows the wireless connection that has been established in two different cells ( $\mathrm{C}$ and $\mathrm{E})$. We can see a big difference in the number of users connecting in both cells. These cells are representative for different areas of the Shopping Center. The cell E covers an area where are located typical common services of a shopping center such as bars, restaurants, bathrooms, information points, etc. For this reason, the number of connected users in this cell is much higher than cell $\mathrm{C}$ has. The cell $\mathrm{C}$ is representative of zones where only a small number of users access the wireless network. 
Table 2.

Power Consumption of different models of APs

\begin{tabular}{lc}
\multicolumn{1}{c}{ AP Model } & PoE (W) \\
\hline Aruba IAP 103 & 9,5 \\
Aruba RAP-100 & 12,5 \\
Cisco Air LAP 1240AG & 15,4 \\
Cisco Aironet 1130AG & 12,2 \\
Cisco Aironet 1230G & 13,0 \\
Aerohive AP121 & 16,8 \\
Aerohive AP230 & 16,8 \\
Aerohive AP330 & 16,8 \\
\hline
\end{tabular}

The cell $\mathrm{E}$ statistics have been taken into account for the validation model, because they represent a best typical scenario and it is the worst case for our SDN system, where more users are connected and more resources are required.

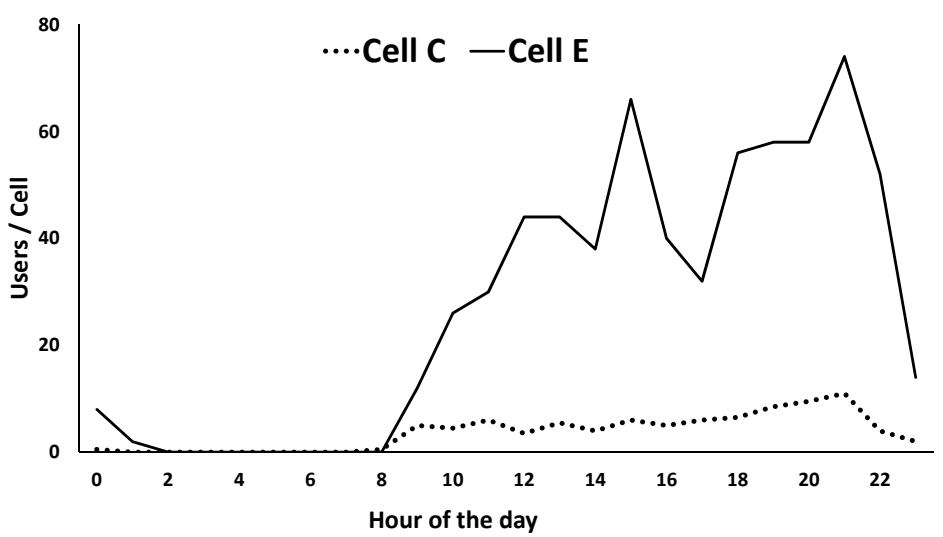

Figure 14.

Users located in Cells $\mathrm{C}$ and $\mathrm{E}$ along the day.

Figure 15 shows the calculation of power consumption of all APs in the topology along one day, when the model uses the cell E user distribution and Aruba IAP 103 APs are utilized. We can see that there is a basis consumption during the hours in which the Shopping Center is closed to the public, from 22:00 to 9:00 hours. From 9:00 hours, when customer begin to get into the shopping center, the requirements of the network resources increase and a new Secondary APs are activated by the SDN system. A decreasing of power consumption is observed from 14:00 to 18:00 hours. This behavior could be explained because regular schedule for lunch starts at 14:00 hours in Spain, where the reference shopping center is located. From 18:00 hours, the amount of customers in the shopping center is increasing, achieving maximum values between 20:00 and 21:00 hours.

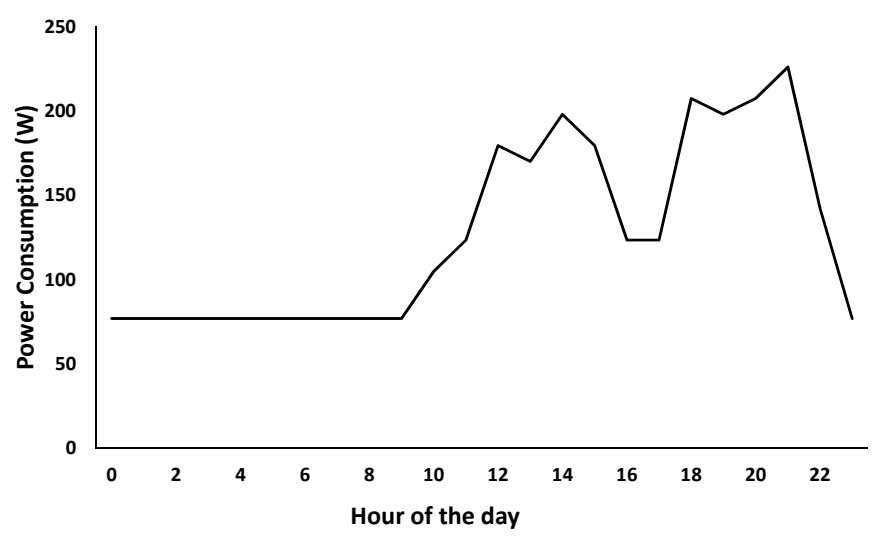

Figure 15. 
Total Power Consumption along the day.

Results of the simulation of the mathematical model related to the saving power are shown in Table 3 . The calculation has been performed based on the power consumption pattern shown in Figure 15 for each model of AP. The second column of the Table 3 (General Power Consumption) shows the total energy consumption for one month, by the whole topology (in KW/h) before to use our proposed SDN system. Then it will correspond with the consumption in real current cases. The calculation has been performed by supposing that the same model of device is used in the whole topology. The third column (Saving Power) shows the power savings obtained when our proposal is applied in the same topologies. We can see that when the SDN solution is implemented we obtain a relevant power saving, approximately $50 \%$.

Table 3.

Comparative on power consumption from different models of APs

\begin{tabular}{lcc}
\hline \multicolumn{1}{c}{ AP Model } & $\begin{array}{c}\text { General Power } \\
\text { Consumption }(\mathbf{K W} / \mathbf{h})\end{array}$ & $\begin{array}{c}\text { Saving Power } \\
(\mathbf{K W} / \mathbf{h})\end{array}$ \\
\hline Aruba IAP 103 & 164,2 & 76,8 \\
Aruba RAP-100 & 216,0 & 101,0 \\
Cisco Air LAP 1240AG & 266,1 & 124,4 \\
Cisco Aironet 1130AG & 210,8 & 98,6 \\
Cisco Aironet 1230G & 224,6 & 105,0 \\
Aerohive AP121 & 290,3 & 135,7 \\
Aerohive AP230 & 290,3 & 135,7 \\
Aerohive AP330 & 290,3 & 135,7 \\
\hline
\end{tabular}

\section{CONCLUSION}

In this paper we have presented a new method to decrease energy consumption in public wireless network using a SDN solution to manage the wireless infrastructure devices, including Ethernet switching and wireless APs. Specifically, in this work we are focused on two functions. First, we have decreased the transmission power of wireless APs, while keeping optimal network performance. The power saving using this option is very low. Second, from this result, a new function has been considered, the activation or deactivation of APs in function of the required resources for the users. This new implementation allows us to reduce the power consumption of the wireless network in a great quantity, approximately $50 \%$.

The proposed solution has been implemented by a new extension of the Openflow protocol over SDN, in order to control dynamically the network devices. The system has been validated by a mathematical model that uses information from real public networks.

In future works, the SDN proposal system will be implemented on three different real networks: a shopping center, a municipal wifi network and a public wifi access network in a mayor museum. Results on power consumption will be compared with data obtained in previous studies before the SDN system would be applied. Additionally, new features and capabilities will be considered to improve the power saving, including artificial intelligence to recognize behaviour patterns and to forecast the power consumption in advance.

\section{ACKNOWLEDGMENT}

This work has been supported by the "Ministerio de Economía y Competitividad", through the "Convocatoria 2014. Proyectos I+D - Programa Estatal de Investigación Científica y Técnica de Excelencia" in the "Subprograma Estatal de Generación de Conocimiento", Project TIN2014-57991-C3-1-P and the "Programa para la Formación de Personal Investigador - (FPI-2015-S2-884)" by the "UniversitatPolitècnica de València".

\section{REFERENCES}

[1] Khoa Nguyen, K., Jaumard, B.(2009) Routing Engine Architecture for Next Generation Routers: Evolutional Trends. Network Protocols and Algorithms 1(1), 62-85

[2] Cisco Visual Networking Index: Global Mobile Data Traffic Forecast Update, 2015-2020 White Paper. Available at: $\mathrm{http}: / / \mathrm{www} . c i s c o . c o m / c / e n /$ us/solutions/collateral/service-provider/visual-networking-index-vni/mobile-white-paper-c11-520862.html. Accessed 3 Nov 2016

[3] Recommended Power Management Settings. Available at https://web.archive.org/web/20071125104605/http://www.climatesaverscomputing.org/tools/pwr_mgmt.html. Accessed 3 Nov 2016

[4] WWF International website. Available at http://wwf.panda.org/. Accessed 3 Nov 2016 
[5] ENERGY STAR. The simple choice for energy efficiency. Available at https://www.energystar.gov/. 3 Nov 2016

[6] Definition WHAT IS SDN?. Available at https://www.opennetworking.org/sdn-resources/sdn-definition. Accessed 3 Nov 2016

[7] OpenFlow. Available at: https://www.opennetworking.org/sdn-resources/openflow. Accessed 3 Nov 2016

[8] McKeown, N., Anderson, T., Balakrishnan, H., Parulkar, G., Peterson, L., Rexford, J., Shenker, S., and Turner, J. (2008) OpenFlow: Enabling Innovation in Campus Networks. ACM SIGCOMM Computer Communication Review, Vol. 38, Issue 2, April 2008, Pp. 69-74.

[9] Dely, P., Kassler, A. and Bayer, N. (2011) OpenFlow for Wireless Mesh Networks. In 2011 Proceedings of 20th International Conference on Computer Communications and Networks (ICCCN), July 31-Aug. 4 2011, Pp. 1-6. DOI: 10.1109/ICCCN.2011.6006100

[10] Guidance document Lot 26 Networked Standby clean FIN.pdf. Available at https://ec.europa.eu/energy/sites/ener/files/documents/Guidance\%20document_Lot\%2026_Networked\%20Standby_clean\%20FIN.pdf . Accessed 3 Nov 2016

[11] Yang, H., Zhang, J., Zhao, Y., Ji, Y., Han, J., Lin, Y., Qiu, Y. L. (2014) Time-aware Software Defined Networking for OpenFlowbased Datacenter Optical Networks, Network Protocols and Algorithms, Vol 6, No 4 (2014). Pp. 77-91

[12] Jimenez, J. M., Romero, O., Rego, A., Dilendra, A., Lloret, J. (2015) Study of Multimedia Delivery over Software Defined Networks, Network Protocols and Algorithms, Vol. 7, No 4 (2015). Pp. 37-62

[13] Jimenez, J. M., Romero, O., Rego, A., Dilendra, A., Lloret, J. (2016) Performance Study of a Software Defined Network Emulator, The Twelfth Advanced International Conference on Telecommunications (AICT 2016), May 22 - 26, 2016 - Valencia, Spain

[14] Mininet An Instant Virtual Network on your Laptop (or other PC). Available at http://mininet.org/. Accessed 3 Nov 2016

[15] Nedevschi, S., Popa, L., Iannaccone, G., Ratnasamy, S., and Wetherall, D. (2008) Reducing network energy consumption via sleeping and rate-adaptation, Proceeding NSDI'08 Proceedings of the 5th USENIX Symposium on Networked Systems Design and Implementation, April 16-18, 2008, San Francisco (USA), Pp. 323-336.

[16] Feeney, L.M., Nilsson, M. (2001) Investigating the Energy Consumption of a Wireless Network Interface in an Ad Hoc Networking Environment. In: Proceedings of the Twentieth Annual Joint Conference of the IEEE Computer and Communications Societies, INFOCOM 2001, Anchorage, Alaska, April 22-26, vol. 3, pp. 1548-1557. IEEE (2001)

[17] Andrade-Morelli, S., Ruiz-Sanchez, E., Granell, E., Lloret, J. (2012) Energy Consumption of Wireless Network Access Points. In Second International Conference, GreeNets 2012, Gandia, Spain, October 25-26, 2012, pp 81-91, DOI 10.1007/978-3-642-37977-2_8

[18] Chen, T., Yang, Y., Zhang, H., Haesik, K., Horneman, K. (2011)Network energy saving technologies for green wireless access networks. IEEE Wireless Communications, Volume 18, Issue 5, pp.30-38.

[19] Tapia, A., Maitland, C., Stone, M.(2006) Making IT work for Municipalities: Buildingmunicipal wireless networks. Government Information Quarterly 23(3), 359-380

[20] van Drunen, R., Koolhaas, J., Schuurmans, H., Vijn, M.(2003) Building a Wireless Community Network in the Netherland. In: USENIX 2003 / Freenix Annual Technical Conference Proceedings, San Antonio, Texas, USA, June 9-14, pp. 219-230

[21] Vu, T. H., Nam, P. N., Thanh, T., Hung, L. T., Van, L. A., Linh, N. D., Thien, T. D., Thanh, N. H.(2012) Power aware OpenFlow switch extension for energy saving in data centers. In International Conference on Advanced Technologies for Communications, ATC 2012, IEEE, Hanoi, Vietnam, 10-12 oct. 2012, pp.309-313, DOI: 10.1109/ATC.2012.6404282

[22] Kaup, F., Melnikowitsch, S., Hausheer, D. (2014) Measuring and Modeling the Power Consumption of OpenFlow Switches, In IEEE International Conference on Network and Service Management (CNSM), Rio de Janeiro, Brazil, 17-21 Nov. 2014, pp. 181-186.

[23] DATA SHEET. ARUBA 103 SERIES ACCESS POINTS. Available at: http://www.arubanetworks.com/assets/ds/DS_AP103Series.pdf. Accessed 3 Nov 2016

[24] DATA SHEET. ARUBA RAP-100 SERIES REMOTE ACCESS POINTS. Available at: http://www.arubanetworks.com/assets/ds/DS_RAP-100.pdf. Accessed 3 Nov 2016

[25] Cisco Aironet 1240AG Series 802.11A/B/G Access Point Data Sheet. Available at: http://www.cisco.com/c/en/us/products/collateral/collaboration-endpoints/unified-ip-phone-7900series/product_data_sheet0900aecd8031c844.html. Accessed 3 Nov 2016

[26] Cisco Aironet 1230AG Series 802.11A/B/G Access Point Data Sheet. Available at: http://www.cisco.com/c/en/us/products/collateral/wireless/aironet-1230-ag-series/product_data_sheet0900aecd801b9068.html. Accessed 3 Nov 2016

[27] Cisco Aironet 1130AG IEEE 802.11 A/B/G Access Point. Available at: http://www.cisco.com/c/en/us/products/collateral/collaboration-endpoints/unified-ip-phone-7900series/product_data_sheet0900aecd8031c844.html Accessed 3 Nov 2016

[28] Aerohive AP121. Available at: http://media.aerohive.com/documents/Aerohive_Datasheet_AP121. Accessed 3 Nov 2016

[29] Aerohive AP230. Available at: http://media.aerohive.com/documents/Aerohive_Datasheet_AP230.pdf. Accessed 3 Nov 2016

[30] Aerohive AP330. Available at: http://media.aerohive.com/documents/Aerohive_Datasheet_AP330.pdf. Accessed 3 Nov 2016 